\title{
ELIMINATING MODALITY FROM THE DETERMINISM DEBATE? MODELS VS. EQUATIONS OF PHYSICAL THEORIES
}

\author{
THOMAS MÜLLER \\ Utrecht University
}

This paper addresses a specific question of reductionism, viz., the question of whether modalities are basic for the notions of determinism and indeterminism, or whether one can do without them. I will argue that the current treatment of these notions within philosophy of science, which takes determinism and indeterminism to be properties of scientific theories rather than metaphysical theses about what the world is like, amounts to a reductionist stance with respect to modality for which no good reasons have been given. Furthermore, I will show that the current implementation of that treatment is not without problems: there is a discrepancy between the official definition of determinism and indeterminism, phrased in terms of the 'modally flat' collection of models of a theory, and the practice of assessing determinism ${ }^{1}$ by looking at the possibly branching space of solutions to a theory's constitutive equations, which moves that practice much closer to a pro-modality stance.

Apart from commenting on use of models vs. equations in the determinism debate within philosophy of science, my paper is also an attempt at getting clear on the proper dialectics of the question of modal reductionism. I will thus also lay out my view as to how determinism and indeterminism or other modal notions should be addressed.

${ }^{1}$ And thus, indeterminism. In what follows I will often mention, for brevity's sake, just one of the notions when I am in fact concerned with both. 
1 Modality and the Metaphysical Question of Determinism Vs. INDETERMINISM

There are two ways to read the question of what the world is like. One, which may be called the everyday reading, points to a question that can be answered by straightforward empirical experiment and observation, e.g., by describing the breeding cycle of a population of penguins living in a particular region, by finding out about the crystal structure of diamonds, or, if you wish, by counting the occurrences of the letter " $r$ " in this paper. As a philosopher, however, one is often concerned with another reading of the question of what the world is like - and one, it seems, that would still be open after all the empirical questions have been answered. This metaphysical sense of the question may be gestured towards by asking what the world is really like. Specific instances of the question of what the world is like taken in that sense are, e.g., about the reality of colour (is the world really such that objects have colour?), or about the nature of time (is there really a distinguished present?). ${ }^{2}$ The question of whether the world is deterministic or not, which is perceived by many to have enormous consequences for our understanding of ourselves as free agents, ${ }^{3}$ is also first and foremost a metaphysical one (and in fact it may be a very close cousin of the question about the nature of time) ${ }^{4}$ determinism and indeterminism are metaphysical notions. They are not about what does or doesn't happen, but about what can or what has to happen and are thus built upon the modal notions of possibility and necessity. Since one can only experience that which is actual and not what is merely possible, empirical research cannot resolve questions of possibility and necessity directly.

It has become common to distinguish a number of different kinds of modality, e.g., to distinguish logical from metaphysical and physical possibility: after all, what is physically impossible (like going faster than the speed of light) may still be metaphysically possible. For the question of determinism, the pertinent notion of possibility is often taken to be physical possibility. This notion of possibility, being tied to the abstract concept of laws of nature, is however too far removed from our initial practical con-

${ }^{2}$ For arguments to the effect that this is how we should understand metaphysics, and for more about these specific examples, cf. Stroud [2000] and Fine [2005, pp.261320].

${ }^{3}$ For an overview of the current state of the free will debate cf., e.g., the articles in Kane [2002].

${ }^{4}$ This connection has been explored, e.g., in the tradition of Prior's [1957] and [1967]. 
cerns about determinism. Those concerns, which provide our first motivation for being interested in the metaphysical notions of determinism and indeterminism, are not connected with abstract laws, but with concrete situations. The appropriate notion of possibility in discussing determinism is, therefore, what has been called real or historical possibility: possibility in a given, concrete situation. ${ }^{5}$

Even if one doesn't subscribe to the thesis of indeterminism, just by asking the question of whether determinism is true of false (and thus, whether indeterminism is false or true) one thus needs to take seriously the notion of real possibility, i.e., of an open possibility in a given concrete situation. My point is not that this proves the existence, or even just the relevance, of real possibility once and for all. But it determines where the dialectics of the argument has to start, since this is how the question, initially, makes sense to us. It may turn out that there are arguments against setting up the discussion in this way. Then, the discussion will have to be transformed. But first and foremost, determinism and indeterminism are based on real possibility.

One open real possibility is enough to prove indeterminism, and thus to disprove determinism. It is however clear from the metaphysical nature of the determinism/indeterminism question as laid out here that there can be no hope of answering that question empirically in any straightforward manner. Possibilities can be experienced only once they are actualized, by which time they have ceased to be open possibilities and have become real in the simple, empirical sense of that term.

This seems to create a problem for the approach to determinism that I am advocating. Determinism is true if and only if there is only one way that the future can (really) turn out to be, whereas indeterminism is true if there is at least one open real possibility. I have suggested that we understand that notion well enough, but I have also said that it is not empirically accessible, at least not in any straightforward sense. But is the question of determinism, on that reading, then really a genuine question and not just nonsense? The importance of the question for our practical lives in my view points strongly to its reality. This link can of course also be questioned. It is true that people are often enough caught up in superstitions,

\footnotetext{
${ }^{5}$ This notion of possibility has not been much discussed outside rather formal contexts; cf. Xu [1997], Belnap et al. [2001] and Placek and Müller [2007]. Historically, however, it is already invoked in Aristotle's famous argument about the sea-battle tomorrow in De Interpretatione.
} 
viewing as practically important questions that cannot be answered and shouldn't even be asked - but this doesn't seem to be one of them. ${ }^{6}$ Furthermore, the reality and importance of the question is corroborated by the fact that we acknowledge modal truths about most, if not all, ordinary things. Chances are you are reading this from a book (do the obvious substitution if you're reading from a different source). It is nothing out of the ordinary to claim that, as of your reading it in the concrete situation you are in, it is really possible that the book should still exist in 100 years' time (in fact you know how to make pretty sure, even if this may be costly and involve steel casing embedded in layers of concrete), and that it is also really possible that it shouldn't (the easier exercise). Real possibilities are nothing spooky or special, we live in a world full of them. This world we picture, at least intuitively, as a branching arrangement of possible histories. $^{7}$

It is a curious fact that amongst philosophers, proponents of the commonsensical view of real modality appear to be a minority, while extreme positions such as Quine's or Lewis's are favored by a majority. ${ }^{8}$ These extreme views are however revisionist, which means that the onus of proof is on their side: in order to adopt one of them one would seem to need good arguments. The general methodological point here is that "you start from where you are", not necessarily "you stay where you are". My further specific point is that in the case of determinism and indeterminism we should also stay where we are, because there aren't any good arguments to pull us away from there.

${ }^{6}$ This is a tangled issue, as anyone who has surveyed the current free will debate will acknowledge. There are numerous writers who try to convince one that the open future image of decision and action is a superstition that should be abandoned in the name of science. My point here is not to enter that debate, but to get clear on the dialectics of the argumentation. Even people who argue against the open future conception of indeterminism acknowledge that we do have such an image of the open future. My point is that, therefore, this is where the discussion should start: with the facts of what Strawson calls "descriptive metaphysics". Of course we could still be wrong - but strong arguments would be needed in order to prove this.

${ }^{7}$ Some people may prefer the "growing block" picture that one finds in Broad [1923]. For present purposes the difference doesn't matter much.

${ }^{8}$ Cf. Fine [2005, pp.214-231]. 


\section{DEFINING DETERMINISM AND INDETERMINISM IN PHILOSOPHY OF SCIENCE}

Above I have already given a rough and ready definition of determinism and indeterminism: determinism is true if and only if there is only one way that the future can (really) turn out to be, whereas indeterminism is true if there is at least one open real possibility. This is not the definition of determinism that is employed in the current discussion about determinism and indeterminism in philosophy of science. There, the focus is, on the contrary, exclusively on scientific - mostly, physical - theories, and determinism is defined as a property of such theories, i.e., of pictures we make of the world, not of the world itself. Roughly, a theory is said to be deterministic if and only if it describes a unique temporal evolution of each of the systems falling under it.

It is easy enough to imagine how it may have come about that the discussion shifted from the metaphysical question to one about properties of theories. (No specific historical claims intended.) Modal notions have poor empiricist credentials (if they have any at all), and current philosophy of science is a brainchild of the logical empiricism of the 1930s. The modalities can be circumvented - or so it seems - by moving the determinism discussion towards a study of physical theories: abstract and therefore modally innocent entities. (Never mind their empiricist credentials.) And it seems clear enough when one should call a specific scientific theory "deterministic" and when "indeterministic", via uniqueness of temporal evolution.

It took some ingenuity to spell out that idea in a succinct fashion. Earman [1986] relates the history and points out the crucial role of Montague [1962], who was the first to advance a model-theoretic definition of the notion of determinism. Basically, a theory is (future-)deterministic ${ }^{9}$ if for any two of its models describing the possible temporal evolution of a system, coincidence of the state at one time brings with it coincidence at all times from then on, where "coincidence" is to be spelled out in terms of a suitable mapping. Montague in fact considered identity of states and pre-

\footnotetext{
${ }^{9}$ Here and in the following we will discuss the notion of future-determinism; there is obviously a temporal mirror image to all the definitions. Sometimes the term "determinism" is used to encompass both past- and future-determinism. Most fundamental physical theories are time reversal invariant and thus future-deterministic if and only if they are also past-deterministic.
} 
supposed a time axis outside the models to be given. In this he is followed by Earman [2007]. Butterfield [2005] in his highly informative Routledge Encyclopedia article on determinism however points out that presupposing such an external meta-time "would be very questionable" 10 and instead bases his definition on a broader class of mappings, viz., isomorphisms "in the usual sense used by logicians". "Accordingly, he gives the following definition:

\section{[A] theory is deterministic if, and only if: for any two of its models, if they have instantaneous slices that are isomorphic, then the corresponding final segments are also isomorphic.}

An instantaneous slice here comprises all the relevant information about the system in question at one point in time - its state at that time -, while the corresponding final segment comprises the whole future development of the state from that time on. Much depends on how much of the system the isomorphism in questions 'sees', or what the instantaneous state of the system - the information about the system available at an instantaneous slice - is taken to be. Obviously, if that state encodes information about the future evolution of the system (think of natural-language predicates such as "mortally wounded"), the system may be judged to be deterministic without good reasons; the same can happen if the state employed is too thin, missing out on crucial information (like calling a quantum system deterministic because total charge is conserved). Butterfield is explicit about the fact that the notion of state at issue does play this crucial role, and that there is no formal way to extract the relevant notion out of a theory - it is rather that considered judgment is called for. Earman [2007] supplants these caveats by pointing to difficulties with the notion of an instantaneous state in various space-time theories. In fact, addressing questions of determinism or indeterminism of theories tends to take one to the heart of con-

\footnotetext{
${ }^{10}$ It surely would be, and it should give rise to extensional differences between, e.g., Earman and Butterfield, if the official model-theoretic definition were actually employed. In section 3.2 below I will point out that this is not so.

${ }^{11}$ In logic, a function $f$ is called an isomorphism between models $A$ and $B$ of a language if $f$ maps the domain of $A$ bijectively onto that of $B$ and carries the respective interpretation along, so that, e.g., $f\left(c_{i}^{A}\right)=c_{i}^{B}$ for a constant symbol $c_{i}$, and $P_{i}^{A}(x)$ if and only if $P_{i}^{B}(f(x))$ for a one-place predicate symbol $P_{i}$. Note that in the discussion of determinism in philosophy of science the language in question is hardly ever made explicit, so that the notion of an isomorphism remains vague.
} 
ceptual issues about these theories, a fact that Earman [2007] rightly emphasizes. Norton additionally points out that questions of determinism also tend to trigger questions of the physicality or otherwise of a model system, such as his famous dome [Norton 2008b].

In section 3, I will look at the actual practice of assessing the determinism of physical theories, i.e., at the way the above definition is put to use in philosophy of science. Before that, however, I would like to comment on the relation between the discussion about the determinism of theories and the primary metaphysical question of determinism addressed in section 1 above.

It is clear that the question about the determinism of theories is not the same as the primary, metaphysical one, and I have suggested that the discussion in terms of properties of theories was chosen intentionally because the other, metaphysical, discussion was viewed with suspicion (or worse). Still, it is that metaphysical notion of determinism that plays a role, or should play a role, e.g., in the free will debate. So what is the point of suggesting, as the use of the same terminology insinuates, that the same issues are addressed?

I see two motivations, both of which are in fact discernible in Butterfield [2005]. First, one may be convinced that the general metaphysical question makes no sense. I have tried to argue that it does make sense, but obviously sentiments differ. If the original metaphysical question makes no sense, it is a good idea to go for the next best thing, and that would indeed be the determinism or indeterminism not of the world, but of pictures we make of the world, e.g., physical theories. A second motivation is that investigating scientific theories may tell us something about the metaphysical question after all. The idea here is that if a scientific theory is deterministic, that means that the world, if the whole truth about it were described by that theory, would also be deterministic; and the same holds for indeterminism. Now since physics strives to give us ever more encompassing theories (some of which have historically been claimed, wrongly, to be the whole truth about the world) and may be hoped to approximate the true nature of our world (for whichever reason), assessing current physics as to determinism seems to be our reasoned best guess at the metaphysical question.

Both these motivational stories strike me as dubious. Against the first I have tried to argue that our methodological starting point needs to be our intuitive self-conception, which would answer the metaphysical question in favor of indeterminism - not as an unassailable fact, but as something 
against which one would need to argue. Saying that the metaphysical question is indeed metaphysical isn't an argument against it; it is just pointing to a possible epistemological problem. The second point strikes me as basically sound - of course we should listen to science. But I believe that the evidence that science gives on the issue of determinism is much richer than what is encoded in scientific theories. Scientific practice and the use of the experimental method seem to me to provide stronger arguments in favor of indeterminism than any specific theory could provide. That practice relies on the possibility of freely choosing initial conditions for experiments. Experiment isn't just observation, but observation after intervention. And intervention is a modal notion: it means to realize a (real) possibility in a concrete situation in which the normal course of things would have been otherwise. If one adjusts a setting of an instrument or pushes a button to start a run of an experiment, that is an intervention; it wouldn't have happened if it hadn't been for the experimenter. Furthermore, if one tries to find out about determinism or indeterminism by looking at currently championed scientific theories, one finds deterministic ones (special relativity theory and maybe some other space-time theories) alongside a number of indeterministic ones (arguably quantum theory, and certainly some spacetime theories as well as Newtonian mechanics). Even for one and the same theory, the verdict as to determinism or indeterminism can depend on fine details of formulation or interpretation [Earman 2007] - determinism (or indeterminism) doesn't seem to be a stable property of a theory that one should expect to be preserved in historical succession. Therefore it does not seem that consensus on the determinism issue is anywhere in sight from the theoretical side. It is rather that the study of scientific theories provides us with toy models of what the world could be like if one such theory were the whole truth about everything, which we know it isn't.

To sum up my points here: I see no good arguments forthcoming that show the infeasibility of investigating a metaphysical notion of determinism and indeterminism. And even if one goes along with the sentiment that the answer to the question of determinism or indeterminism can only come from science, that does not prove that one should only look at scientific theories. In fact there are good reasons for not doing that. First, science is a human practice, and modern science relies on real possibilities in its central notion of experiment. And second, no consensus about determinism or indeterminism of current science - even of current physics - seems to be forthcoming. 


\section{PHySICAL THEORIES: MOdELS VS. EQUATIONS}

The upshot of the above discussion seems to be the following: When it comes to assessing determinism, there are two camps disagreeing about whether the question makes sense as a metaphysical question about what the world is really like, or not. If one thinks that the metaphysical question does make sense, one's project would seem to be that of getting clear about our everyday conception of indeterminism and its role in agency, causation, and scientific practice. If one disagrees and holds the metaphysical question to be nonsensical - for which, as I stressed, a good argument would need to be given that I don't see to be forthcoming, but the discussion is tangled - then what one should do is to investigate, one after the other, specific scientific theories, and assess them as to their determinism and indeterminism; without much hope of arriving at a uniform verdict as to determinism or indeterminism viewed globally. And that is what is happening in philosophy of science, at a very high level of technical sophistication; witness again Earman [2007]: determinism and indeterminism are studied as properties of physical theories.

\subsection{Physical Theories}

It is not so easy to say what a physical theory is - physical theories can be viewed in many ways. According to the well established if somewhat tiresome opposition between a syntactic and a semantic view of theories, a theory can be specified syntactically, as a set of sentences in a specified (traditionally, logically regimented) language - or semantically, as a class of models, abstract mathematical structures. On both views the empirical significance of a theory thus specified has to be spelled out in a further step, commonly by labeling some part of the vocabulary as "observational" or by specifying a notion of isomorphism with structures having direct empirical significance.

If one looks at textbook accounts of physical theories or the way such theories are taught to students, one may wonder which form of representation one is witnessing. Surely a textbook contains a text with a syntactic structure, not an abstract mathematical object. But theories in such textbooks are hardly, if ever, formalized; they do not accord with the ideal of the syntactic view. Nor does one normally find in a textbook a method for constructing abstract classes of models. One does, however, often find something pretty close: Most theories are presented and studied via their 
constitutive equations, and solutions to these equations correspond to models of the theory in question. Thus, a semantic representation of a given physical theory can be obtained as the set of all solutions to the theory's constitutive equations. Such a set normally has a natural subdivision corresponding to the values of specific parameters like mass or charge. Initial values can also function as parameters.

In this way, a theory's constitutive equations determine its models as the space of their solutions.

\subsection{Models vs. Equations in Assessing Determinism}

The official definition of determinism agreed upon in current philosophy of science is the model-theoretic one expressed in the quote from Butterfield [2005] above: a theory is (future) deterministic if for any two of its models that have isomorphic instantaneous slices, the corresponding final segments are also isomorphic. This definition has a precise mathematical meaning. ${ }^{12}$ It all boils down to investigating the separate models expressing the individual time courses of systems allowed by the theory. As such, this approach is completely modality-free: ${ }^{13}$ give me the collection of models extensional, set-theoretic structures -, and I give you back a verdict as to determinism or indeterminism. It may not be easy, and no verdict may be forthcoming in complicated cases (it is not claimed that there is a decision procedure that one could use), but if a verdict is forthcoming, this is how it works.

When one looks at what people actually do when they apparently implement the mentioned definition and check whether a specific theory is deterministic or not, however, the picture changes. Straightforward uses of the model-theoretically phrased definition in the philosophy of science lit-

\footnotetext{
${ }^{12}$ At least once the language of the theory has been described in sufficient detail, cf. note 11. A further point is that the precise meaning that that definition has may not be the intended meaning - there is a subtle issue about the quantification over isomorphisms ("isomorphic" is defined in terms of "there is an isomorphism ...") that also needs to be addressed; see below.

${ }^{13}$ At least if one discounts the remnants of modality in the phrase "allowed by the theory" as a mere metaphor which is to be resolved by extensionally quantifying over all mathematically existing models, without taking recourse to a notion of physicality. Again, this is a tangled issue; cf., e.g., the discussion about our reasons for preferring the retarded solutions to Maxwell's equations, as in Frisch [2008] vs. Norton [2008a], or the discussion of physicality in connection with Norton's dome [Norton 2008b].
} 
erature are hard to find. Instead, it is common practice to look at the theory's defining equations and take recourse to results from mathematical physics that state whether, and under which circumstances, these equations have a unique solution. If these equations guarantee uniqueness of the solution for given initial values, the theory is declared to be deterministic, totally in accordance with the intuition behind calling a theory deterministic if it describes unique time courses of development for systems falling under it. This also makes practical sense: For the assessment of equations in that respect, mathematical physics has provided a large range of techniques and pertinent results, ${ }^{14}$ there are no, or hardly any, comparable results phrased model-theoretically. Thus, studying the equations gives one a fair chance of answering the question of determinism vs. indeterminism of a specific physical theory. Furthermore, it seems that what one is doing is really just the same thing as what is demanded by the official definition, so that the move from models and isomorphisms to studying the equations is completely innocent. The move is made in passing, and no need for justification seems to be felt by those who make it.

The important question is whether this discrepancy between definition and method of assessment really makes no difference. It obviously wouldn't if the equivalence of the two approaches could be demonstrated, so that one would just be a reformulation of the other. And the equivalence is obviously presupposed by researchers working in the field. But whether it really holds depends on fine details that would need to be spelled out. In order to fix the notion of an isomorphism, the language of the theory would have to be made precise (cf. note 11 above), which hardly ever happens. And there is the further question of the intended type of quantification over isomorphisms (cf. note 12 above): is the definition to quantify over isomorphisms existentially (signaling determinism if for any isomorphic instantaneous slices there is some isomorphism identifying the final seg-

\footnotetext{
${ }^{14}$ Some of these results are technically quite involved; cf., e.g., Xia [1992] on the collision-free five-body problem in classical mechanics. As a simpler example, for differential equations, the typical form of constitutive equations of dynamical theories, it is often sufficient to study some structural properties in order to find out whether unique solutions are guaranteed. A case in point is the Picard-Lindelöf theorem stating that the initial value problem $d x(t) / d t=f(t, x(t))$ for given $x\left(t_{0}\right)$ has a unique solution around $t_{0}$ if $f$ obeys certain conditions (Lipschitz continuity in $x$, continuity in $t$ ). Conversely, violations of these conditions allow for indeterministic time development. Norton's dome [Norton 2008b] is built on exactly this strategy.
} 
ments) or universally (demanding agreement for all such isomorphisms), and may different isomorphisms be employed? To make these questions a little more precise, let $A$ and $B$ stand for two models of a theory $T$, let $A_{t}$ and $B_{t^{\prime}}$ be instantaneous slices (where the labels " $t$ " and " $t$ " " do not belong to the models themselves, nor to a meta-time, as stressed by Butterfield), and let $A_{>t}$ and $\mathrm{B}_{>_{t}}$ be the corresponding final segments. Then the official definition clearly needs to be spelled out as follows:

$T$ is deterministic if, and only if: for any models $A$ and $B$ of $T$ and for all their instantaneous slices $A_{t}$ and $B_{t^{\prime}}$, if there is an isomorphism $f$ between $A_{t}$ and $B_{t}$, then there is an isomorphism $g$ between $A_{>t}$ and $B_{>}$.

Thus, different isomorphisms may be used to identify the slices and the final segments. But shouldn't one demand that the same isomorphism $\mathrm{f}$ that identifies the instantaneous slices, also identifies the final segments? Furthermore, the quantification is existential, but it is not clear that this always gives the correct assessment. Thus, in a toy theory of discrete time radioactive decay pictured as a branching tree in Figure 1, in (A) an isomorphism identifying $\mathrm{m}_{\mathrm{o}}$ in history (model) $\mathrm{h}_{0}$ with $\mathrm{m}_{1}$ in history (model) $\mathrm{h}_{1}$ can be extended to an isomorphism for the corresponding final segments (the end points of the respective histories) and thus threatens to signal determinism for the obviously indeterministic scenario. In (B) this even holds if one includes the whole past history of the system. In these cases, the overall assessment remains correct because the definition also forces one to look at the isomorphism between $\mathrm{m}_{0}$ in $\mathrm{h}_{0}$ and $\mathrm{m}_{0}$ in $\mathrm{h}_{1}$, for which no isomorphism between the corresponding final segments can be given. In a modified deterministic scenario of (B) with just the one history $h_{1}$, the assessment however becomes incorrect, as the isomorphism between $\mathrm{m}_{0}$ and $\mathrm{m}_{1}$ isn't accompanied by a corresponding isomorphism between the corresponding final segments and the definition thus signals indeterminism.

Obviously many responses are possible with respect to these toy models - e.g., I haven't specified a language either, nor shown that there is any serious physical content behind my pictures. I deliberately called them toy models. My point is just that if the official definition of determinism in terms of models and isomorphisms were actually employed, such cases, and more elaborate ones like them closer to actual physics, would have to be scrutinized in detail in order to check the tenability of the definition. This however does not happen - and since the question of determinism or indeterminism is really approached from the point of view of a theory's 
equations, these questions do not need to be answered, and no confusion threatens. A proper mathematical study of the equations is enough, there is sufficient context to identify cases in which the same state can be followed by different time courses of development.

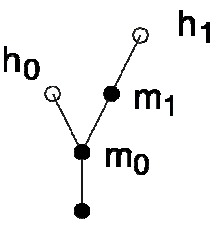

A

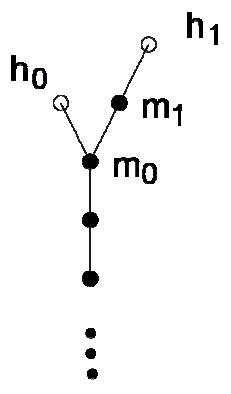

B

Figure 1: Toy model for radioactive decay in discrete time. Open circles indicate that the particle has decayed. (A) First time step specified, (B) backwards infinite time. See text for details.

\subsection{Consequences for the Role of Modality in the Determinism Debate}

Given that there is such a large discrepancy between official definition and actual practice of assessment when it comes to the determinism or indeterminism of theories, one may wonder how this situation could arise and why it is sustained. I will end this paper by trying to spell out my - rather speculative - diagnosis of this situation.

Historically, the model-theoretic tradition in which Montague worked certainly had an enormous and also a very beneficial influence on the discussion of the determinism of theories. Montague [1962] points out that the determinism debate may be trivialized unless one pays attention to a large number of fine details. The definition in terms of models and isomorphisms however seems to me to be preferred also for different, metaphysical reasons: it is extremely well suited to the general metaphysical outlook propounded by Lewis [1986]. ${ }^{15}$ According to Lewis, modality needs to be understood not as something inherent in the world we live in, but rather as based on counterpart relations between wholly separate and equally real "worlds". Thus, e.g., no two worlds can contain the same individual. In Figure 1 I pictured the time development of systems in terms of branching histories, i.e., a single past time development giving rise to different possi-

\footnotetext{
${ }^{15}$ Lewis is explicitly invoked, e.g., in Earman [2007].
} 
ble future developments. This is in accord with what happens when one studies the time development of a system's state according to constitutive equations, and it is very close to the intuitive understanding of indeterminism as involving open real possibilities. But it is not in accord with a Lewisian outlook on modality, which demands wholly separate worlds between which isomorphisms may act as a counterpart relation.

The discrepancy between a model-theoretic definition of determinism and its assessment via a study of constitutive equations is thus interpretationally highly significant. Models are abstract and extensional creatures; quantifying over them, or over isomorphisms between them, can never amount to building up real modality. Giving a model-theoretic definition of determinism and indeterminism is thus in full accord with the project of reducing modalities away. The practice of assessing determinism via a study of constitutive equations however does not support this stance. Thus, even practitioners of an officially model-theoretic approach to determinism and indeterminism in philosophy of science should acknowledge the importance of real modality.

\section{CONCLUSIONS}

In this paper I have tried to argue that it makes good sense to view the question of determinism vs. indeterminism in the way in which that question initially presents itself to us: As a practically and theoretically relevant question about what the world is really like. Despite some epistemological worries, the notion of modality that I identified as lying behind the distinction between determinism and indeterminism, real modality, is sufficiently well understood.

This picture is apparently threatened by a manner of treating the issue of determinism vs. indeterminism that has strong currency in philosophy of science, and which seems to give a strong argument for the elimination of real modality from the determinism debate: As defined in philosophy of science, determinism and indeterminism are simply properties of physical theories that can be assessed purely extensionally, via a study of a theory's models. My reaction to this threat has been twofold. First, while agreeing with the slogan "go scientific", I have stressed that science as a human endeavor is more than just the production of abstract structures: it involves intervention in the course of nature via experiment, and real possibilities lie at the bottom of that practice. Second, I have tried to show that 
the modality-unfriendly outlook of the study of determinism in philosophy of science is at odds with the very method employed in that field: the model-theoretic definition is not implemented, but rather given up in favor of a much more modality-friendly way of looking at things, viz., the study of constitutive equations and the possibly branching space of their solutions. The diversity of results obtained by such studies furthermore strengthens my first reaction: given that no unique verdict as to determinism or indeterminism of scientific theories in general seems to be forthcoming, the impact of science on the question of determinism can only be weighed by considering the practice of science as a whole, not just one of the products of that practice.

Thus, science and the philosophical study of science do not pose a threat to the importance of real modality for the metaphysical question of determinism, nor do they provide arguments for a reductionist stance with respect to real modality.

\section{ACKNOWLEDGEMENTS}

I would like to thank the organizers of and my audience at the 31st International Wittgenstein Symposium, Kirchberg, 13 August 2008. Thanks to Janneke van Lith and Sebastian Lutz for helpful comments on a previous draft. Support by the Deutsche Forschungsgemeinschaft is gratefully acknowledged.

\section{REFERENCES}

Belnap, N., Perloff, M., and Xu, M. [2001]. Facing the Future. Agents and Choices in Our Indeterminist World. Oxford: Oxford University Press.

Broad, C.D. [1923]. Scientific Thought. New York: Harcourt, Brace and Co.

Butterfield, J. [2005]. Determinism and Indeterminism. In Craig, E., editor, Routledge Encyclopedia of Philosophy. London: Routledge. Retrieved March 7, 2008, from http://www.rep.routledge.com/article/Q025.

Earman, J. [1986]. A Primer on Determinism. Dordrecht: Reidel.

Earman, J. [2007]. Aspects of Determinism in Modern Physics. In Butterfield, J. and Earman, J., editors, Handbook of the Philosophy of Physics, pp.1369-1434. Amsterdam: Elsevier.

Fine, K. [2005]. Modality and Tense. Oxford: Oxford University Press.

Frisch, M. [2008]. Causal Reasoning in Physics. Available at http://philsci-archive.pitt. edu/archive/00003820/. 
Kane, R., editor [2002]. The Oxford Handbook of Free Will. Oxford: Oxford University Press.

Lewis, D. [1986]. On the Plurality of Worlds. Oxford: Blackwell.

Montague, R. [1962]. Deterministic Theories. In Willner, D., editor, Decisions, Values and Groups, pp.325-370. Oxford: Pergamon Press. Reprinted in Formal Philosophy, ed. R.H. Thomason, New Haven, CT: Yale University Press 1974, pp.303-359.

Norton, J. [2008a]. Is There an Independent Principle of Causality in Physics? A Comment on Mathias Frisch, "Causal Reasoning in Physics.". Available at http://philsci-archive.pitt.edu/archive/00003832/.

Norton, J. [2008b]. The Dome: An Unexpectedly Simple Failure of Determinism. Philosophy of Science 75(5), pp.786-798.

Placek, T. and Müller, T. [2007]. Counterfactuals and Historical Possibility. Synthese, 154: pp.173-197.

Prior, A.N. [1957]. Time and Modality. Oxford: Oxford University Press.

Prior, A.N. [1967]. Past, Present and Future. Oxford: Oxford University Press.

Stroud, B. [2000]. The Quest for Reality: Subjectivism and the Metaphysics of Colour. Oxford: Oxford University Press.

Xia, Z. [1992]. The Existence of Noncollision Singulatities in the $n$-body Problem. Annals of Mathematics, 135: pp.411-468.

Xu, M. [1997]. Causation in Branching Time (I): Transitions, Events and Causes. Synthese, 112(2): pp.137-192. 